BULLETIN (New Series) OF THE

AMERICAN MATHEMATICAL SOCIETY

Volume 36, Number 4, Pages 523-527

S 0273-0979(99)00793-4

Article electronically published on July 27, 1999

Geometry of subanalytic and semialgebraic sets, by M. Shiota, Birkhäuser, Boston, MA, 1997, xii+431 pp., \$89.50, ISBN 0-8176-4000-2

\title{
General PRESENTATiON
}

This book presents an axiomatic treatment of subanalytic geometry, which can be applied to other classes of subsets of Euclidean spaces. Namely, Shiota considers a class $\mathfrak{X}$ of subsets of Euclidean spaces satisfying the following axioms:

(i) Every algebraic subset of $\mathbb{R}^{n}$ is $\mathfrak{X}$.

(ii) If $X_{1} \subset \mathbb{R}^{n}$ and $X_{2} \subset \mathbb{R}^{n}$ are $\mathfrak{X}$, then $X_{1} \cap X_{2}, X_{1} \backslash X_{2}$ and $X_{1} \times X_{2}$ are $\mathfrak{X}$.

(iii) If $X \subset \mathbb{R}^{n}$ is $\mathfrak{X}$ and $p: \mathbb{R}^{n} \rightarrow \mathbb{R}^{m}$ is a linear map such that the restriction of $p$ to the closure of $X$ is proper, then $p(X)$ is $\mathfrak{X}$.

(iv) If $X \subset \mathbb{R}$ is $\mathfrak{X}$, then each point of $X$ has a neighborhood in $X$ which is a finite union of points and intervals.

The two most widely studied examples are the classes of semialgebraic sets and of subanalytic sets. The former is the smallest class satisfying (i), (ii) and (iii) (which does, indeed, turn out to satisfy (iv) by a famous theorem of Tarski), while the latter is defined similarly except that we replace "algebraic" by "semianalytic" in (i). (A set is called semianalytic if it can be described locally by equalities and inequalities between real analytic functions.) The fact that (iv) is satisfied in this case is due to Gabrielov. These two classes have formed the foundations for the study of semialgebraic geometry (an important topic in real algebraic geometry) and real analytic geometry respectively. It is Shiota's aim to recover from the above axioms alone most known properties of subanalytic sets (one loses Łojasiewicz inequality in its usual form, and one has to work with $\mathcal{C}^{r}$ differentiability, $r$ positive integer, instead of analyticity). The main interest of the book is that it contains very deep results, some of which are new even for subanalytic or semialgebraic sets. Among the most important results are the $\mathfrak{X}$-versions of Thom's transversality theorem and Thom's isotopy lemmas (basic tools for developing a "tame" or $\mathfrak{X}$-equisingularity theory) and the uniqueness of $\mathfrak{X}$-triangulations (which shows that $\mathfrak{X}$-topology is close to PL-topology).

The axioms for a class $\mathfrak{X}$ are very close to the definition of an o-minimal structure expanding the field $\mathbb{R}$ (as presented in the recent book by van den Dries [vdD]). One gets an o-minimal structure by removing the properness assumption in (iii) and replacing (iv) by its global counterpart: If $X \subset \mathbb{R}$ is $\mathfrak{X}$, then $X$ is a finite union of points and intervals. Such an o-minimal structure is called a class $\mathfrak{X}_{0}$ by Shiota. The interesting examples of classes $\mathfrak{X}$ beyond the subanalytic sets are given by the structures which were proved to be o-minimal by Wilkie and others [Wi1], [Wi2]. Shiota claims that the notion of a class $\mathfrak{X}$ covers more situations, taking the example of subanalytic sets which form a class $\mathfrak{X}$, but not an o-minimal structure. On the other hand, it is explained in [vdDMi] that o-minimal structures can also treat the subanalytic case, by considering the "global" subanalytic sets, which are also subanalytic at infinity. More precisely and more generally, given a class $\mathfrak{X}$,

1991 Mathematics Subject Classification. Primary 32B20, 14P10, 57Q25, 57R05, 58A35. 
one gets an o-minimal structure by considering $\mathfrak{X}$-subsets of $\mathbb{R}^{n}$ which are also $\mathfrak{X}$ subsets of the projective space $P^{n}(\mathbb{R})$ (recall that $P^{n}(\mathbb{R})$ can be embedded as an algebraic subset of a Euclidean space). In the other direction, given any o-minimal expansion of the field $\mathbb{R}$, one gets a class $\mathfrak{X}$ larger than the class of definable sets by taking all subsets which are locally definable at each point of a Euclidean space. Starting with globally subanalytic sets, one recovers in this way all subanalytic sets. However, the exact relation between the two frameworks is not completely clear: for instance, it seems that the triangulation of subanalytic functions in the noncompact case does not reduce easily to the o-minimal situation. Many results in the $\mathfrak{X}$ framework need assumptions of boundedness and/or the assumption that the class $\mathfrak{X}$ is local (i.e. a subset of $\mathbb{R}^{n}$ is $\mathfrak{X}$ if it is locally $\mathfrak{X}$ at each point of $\mathbb{R}^{n}$ ). Another point is that some proofs in the book are not elementary (in the modeltheoretic sense), because they rely on specific properties of the reals such as local compactness or the archimedean property. This is the case, for instance, for the proof of the triangulation of functions. Elementary proofs are important because they give very easily results of trivialization and finiteness (cf. [vdD] chap. 8, 2.13, or $[\mathrm{Co}])$. It would be interesting to extend Shiota's results to o-minimal structures expanding a real closed field.

The reading of Shiota's book is not easy. Several proofs are very long and require deep concentration. The reader has to supply his own figures in order to understand the situations and memorize the notation (there are only two figures in the book). Many of the technical difficulties seem to be inherent to the results which are obtained. These results are very important and provide foundations for the development of a "tame topology" and a "tame singularity theory". Shiota's book is indispensable to every mathematician interested in these topics.

\section{DESCRIPTION OF THE CONTENT}

Chapter 1. The first section of Chapter 1 contains a review of Whitney stratifications. It introduces two notions which play important roles in the following:

1) The notion of a removal data, which defines a system of tubular neighborhoods of strata (of radii smaller and smaller as the dimension of strata increases) such that, if one removes from a stratum $X_{i}$ the intersection of $X_{i}$ with such neighborhoods of strata $X_{j}$ contained in the closure of $X_{i}$, the remaining part is a manifold with corners.

2) The notion of friendly functions. Two functions are called friendly if their gradients never vanish or point in opposite directions.

The notion of friendliness allows one to define strong isomorphisms $f: X \rightarrow Y$ between Whitney stratified sets by imposing some friendliness condition on the functions measuring the distance to strata $X_{i}$ and $f\left(X_{i}\right)$. A strong isomorphism $f: X \rightarrow Y$ implies that the remaining part of $X_{i}$ after removal given by a removal data is $\mathcal{C}^{\infty}$-diffeomorphic to the remaining part of $f\left(X_{i}\right)$.

The second section of Chapter 1 is a brief tour of subanalytic and semialgebraic geometry. It is observed that two nonnegative continuous subanalytic functions with the same zero-set $Z$ are friendly in a neighborhood of $Z$. Hence, subanalytic isomorphisms between subanalytic Whitney stratified sets are always strong.

The third section begins with some facts of PL-topology. Shiota introduces cell complexes, different from the usual ones, in order to make possible finite cell triangulations in noncompact situations. 
The end of this section is devoted to $\mathcal{C}^{\infty}$ triangulations. Following the exposition by Munkres of the Cairn-Whitehead theorem on unique $\mathcal{C}^{\infty}$ triangulability of manifolds $[\mathrm{Mu}]$, Shiota proves that a subset of $\mathbb{R}^{n}$ which is locally $\mathcal{C}^{\infty}$ equivalent to a polyhedron is uniquely $\mathcal{C}^{\infty}$ triangulable. This result is applied to the "remaining after removal" parts of strata of compact Whitney stratified sets. This, in turn, is used to construct a triangulation of a compact Whitney stratified set, with the property that the retractions of neighborhoods of strata become PL maps in the triangulation.

Chapter 2. Chapter 2 is the core of the book. The first section deals with the basic properties of $\mathfrak{X}$-sets, which are similar to the properties of subanalytic sets (the arguments for the first results present similarities with those of [PiSt], [KnPiSt], but they are shorter because they use specific properties of $\mathbb{R}$ ). In particular, it is proved that $\mathfrak{X}$-sets and maps have canonical $\mathcal{C}^{r}$ Whitney stratifications ( $r$ a positive integer). Unlike the subanalytic case, it is not known in general that they admit analytic stratifications. Another result is the friendliness of $\mathfrak{X}$-functions. In the second section it is proved that $\mathfrak{X}$-sets are triangulable, with a triangulation homeomorphism which is of class $\mathfrak{X}$. The proof follows a classical pattern and relies on a "good projection" lemma.

The third section is devoted to the triangulation of $\mathfrak{X}$-functions (with values in $\mathbb{R}$ ). The proof assumes the uniqueness of $\mathfrak{X}$-triangulation of $\mathfrak{X}$-sets, which is proved in Chapter 4. The reason is that the choice of a good projection is made locally (with respect to the target $\mathbb{R}$ ), and then uniqueness is needed to glue partial triangulations. In the fourth section it is recalled that, in the semialgebraic case, there is a trick which avoids uniqueness and gluing (actually, this trick can be extended to the o-minimal case; see [Co]). This section also contains the proof of finite "cell triangulation" (cf. Chap. 1, Sec. 3) in the noncompact case, in the semialgebraic or $\mathfrak{X}_{0}$ framework.

The fifth section introduces a topology on the space of $\mathcal{C}^{r} \mathfrak{X}$-maps $(r$ a positive integer). The main result of the section is a transversality theorem giving sufficient conditions for $\mathcal{C}^{r} \mathfrak{X}$-maps whose $r^{\prime}$-jets $\left(r^{\prime}<r\right)$ are transversal to a given stratification of the jet space to be dense. It is also proved that $\mathcal{C}^{r+1} \mathfrak{X}$-maps are dense in the space of $\mathcal{C}^{r}$ ones and that a $\mathcal{C}^{r} \mathfrak{X}$-submanifold of $\mathbb{R}^{n}$ can be approximated by $\mathcal{C}^{r+1}$ ones. Also, a $\mathcal{C}^{r} \mathfrak{X}$-submanifold of $\mathbb{R}^{n}$ can be uniquely compactified as a $\mathfrak{X}$-submanifold with boundary. Note that, in the semialgebraic case, one has approximation by Nash (i.e. analytic semialgebraic) maps; this is a very important tool in the study of Nash mappings and manifolds [Sh1], which has no counterpart in the $\mathfrak{X}$ framework.

The sixth section contains the most important results of this chapter, namely the $\mathfrak{X}$-versions of Thom's isotopy lemmas. Let us state the $\mathfrak{X}$-version of Thom's second isotopy lemma. A diagram

$$
X \stackrel{f}{\longrightarrow} Y \stackrel{p}{\longrightarrow} P
$$

of $\mathfrak{X}$-sets and maps is said to be $\mathfrak{X}$-trivial if there exist $a \in P$ and $\mathfrak{X}$-homeomorphisms $g:(p \circ f)^{-1}(a) \times P \rightarrow X$ and $h: p^{-1}(a) \times P \rightarrow Y$ such that the diagram 


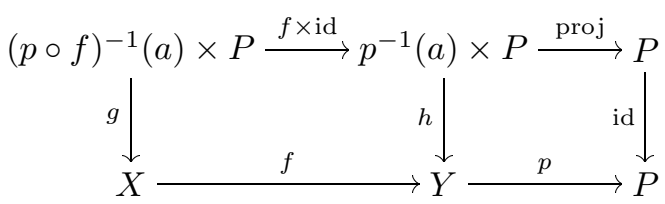

commutes. If $f$ and $p$ are proper $\mathcal{C}^{1} \mathfrak{X}$-maps, $P$ is a contractible $\mathcal{C}^{1} \mathfrak{X}$-submanifold, and $f$ admits a finite $\mathcal{C}^{1}$ Whitney $\mathfrak{X}$-stratification "sans éclatement" such that the restriction of $p$ to each stratum of $Y$ is a submersion onto $P$, then $X \rightarrow Y \rightarrow P$ is $\mathfrak{X}$-trivial.

Since the trivialization homeomorphisms are required to be of class $\mathfrak{X}$, the usual method of integration of vector fields (see [Gial]) cannot be applied. One has to prove first a theorem of $\mathfrak{X}$-triviality of proper $\mathfrak{X}$-submersions which replaces integration of vector fields. The $\mathfrak{X}$-version of Thom's second isotopy lemma is new even in the semialgebraic case.

The last section of the chapter uses the results previously obtained for the investigation of equivalence relations between $\mathfrak{X}$-sets or functions. For instance, two $\mathfrak{X}$-functions $f_{1}: X_{1} \rightarrow \mathbb{R}$ and $f_{2}: X_{2} \rightarrow \mathbb{R}$ are said to be $\mathfrak{X}$-equivalent if there is an $\mathfrak{X}$-homeomorphism $\tau: X_{1} \rightarrow X_{2}$ such that $f_{1}=f_{2} \circ \tau$. It is proved that $\mathfrak{X}$-equivalence is strictly stronger than $\mathcal{C}^{0}$ equivalence and implied by $\mathcal{C}^{1}$ equivalence. The main tool is a theorem of uniqueness of controlled $\mathfrak{X}$-tube systems for $\mathfrak{X}$-stratifications whose strata are $\mathfrak{X}$-diffeomorphic to open balls; the friendliness of $\mathfrak{X}$-functions is crucial here.

Chapter 3. The third chapter is entirely devoted to the "Hauptvermutung", i.e. uniqueness of $\mathfrak{X}$-triangulation: two $\mathfrak{X}$-homeomorphic compact polyhedra in $\mathbb{R}^{n}$ are PL homeomorphic. Shiota conjectures that two compact polyhedra which have strongly isomorphic Whitney stratifications are PL homeomorphic. But he can prove this conjecture only with extra assumptions which strengthen the condition of strong isomorphism. The proof uses the tools presented in the first chapter: $\mathcal{C}^{\infty}$ triangulations, etc. The $\mathfrak{X}$ framework is used only to show that a $\mathfrak{X}$-homeomorphism of compact polyhedra implies a strong isomorphism with the needed extra conditions.

Chapter 4. The fourth chapter presents results on triangulation of $\mathfrak{X}$-maps. Contrary to $\mathfrak{X}$-functions, $\mathfrak{X}$-maps are not always triangulable. For instance, the blow-up map of a point in a plane is not triangulable. A conjecture of Thom states that a proper map which admits a stratification "sans éclatement" is triangulable. Shiota proves the $\mathfrak{X}$-version of this conjecture in the special case where the dimension of every stratum in the source exceeds at most by one the dimension of its image. There are also theorems which describe sufficient and essentially necessary conditions for an $\mathfrak{X}$-map to be $\mathfrak{X}$-triangulable, in terms of what Shiota calls "resolutions" of the map. This notion of resolution can be seen as a wide elaboration on the remark that proper finite-to-one $\mathfrak{X}$-maps are $\mathfrak{X}$-triangulable.

The results on triangulability of maps contained in this chapter are superseded by the recent proof of Thom's conjecture by Shiota [Sh2]. The arguments of this proof are relatively simpler than the arguments of the results in this chapter and rely on tools presented in chapter 1. 
There are also in this chapter remarks on uniqueness of $\mathfrak{X}$-triangulability of $\mathfrak{X}$ maps and the relation with the conjecture that local (with respect to the target) $\mathfrak{X}$ triangulability implies global $\mathfrak{X}$-triangulability. Finally, it is proved that a complex analytic function has a subanalytic triangulation.

Chapter 5. In this last chapter Shiota studies classes $\mathfrak{Y}$ which satisfy the same axioms as $\mathfrak{X}$, except for (i), which is replaced by the weaker

$\left(i^{\prime}\right)$ All rational semilinear (i.e. defined by linear inequalities with rational coefficients) subsets of Euclidean spaces are $\mathfrak{Y}$.

The first section treats the case where every $\mathfrak{Y}$-set is locally semilinear. In the second section, it is shown that, if there exists a $\mathfrak{Y}$-set which is not locally linear and the class $\mathfrak{Y}$ is local, then it reduces to the $\mathfrak{X}$ case via a bijection of $\mathbb{R}$. This last result is related to the "Trichotomy Theorem" of [PeSt].

\section{REFERENCES}

[Co] M. Coste: Topological Types of Fewnomials. Singularities Symposium - Łojasiewicz 70. Banach Center Pub. 44, Polish Acad. Sci. (1998), 81-92. CMP 99:09

[vdD] L. van den Dries: Tame Topology and O-minimal Structures. London Math. Soc. Lect. Notes 248. Cambridge Univ. Press 1998. CMP 98:15

[vdDMi] L. van den Dries, C. Miller: Geometric Categories and O-minimal Structures. Duke Math. J. 84 (1996), 497-540. MR 97i:32008

[Gial] C.G. Gibson et al.: Topological Stability of Smooth Mappings. Lecture Notes in Math. 552. Springer-Verlag 1976. MR 55:9151

[KnPiSt] J.F. Knight, A. Pillay, C. Steinhorn: Definable Sets in Ordered Structures. II. Trans. Amer. Math. Soc. 295 (1986), 563-605. MR 88b:03050b

[Mu J.R. Munkres: Elementary Differential Topology. Ann. of Math. Studies. Princeton Univ. Press 1963. MR 29:623

[PeSt] Y. Peterzil, S. Starchenko: A Trichotomy Theorem for O-minimal Structures. Proc. London Math. Soc. 77 (1998), 481-523. CMP 99:01

[PiSt] A. Pillay, C. Steinhorn: Definable Sets in Ordered Structures. I. Trans. Amer. Math. Soc. 295 (1986), 565-592. MR 88b:03050a

[Sh1] M. Shiota: Nash Manifolds. Lect. Notes Math. 1269. Springer-Verlag 1987. MR 89b:58011

[Sh2] M. Shiota: Thom's Conjecture on Triangulation of Maps. Sûrikaisekikenkyûsho Kôkyûroku No. 1050 (1998), 12-28. CMP 99:09

[Wi1] A. Wilkie: Model Completeness Results for Expansions of the Real Field by Restricted Pfaffian Functions and the Exponential Function. J. Amer. Math. Soc. 9 (1996), 10511094. MR 98j:03052

[Wi2] A. Wilkie: A General Theorem of the Complement and Some New O-minimal Structures. Preprint, 1996.

Michel Coste

UNIVERSITY OF RENNES

E-mail address: coste@univ-rennes1.fr 